\title{
Literaturverzeichnis.
}

Beck, P.: Über das schweizerische und europäische Pliozän und Pleistozän. - Eclog. geol. Helv. 1933. - Vorläufige Mitteilung über eine Revision des alpinen Quartärs. - Ebendort 1937.

Bräuhäuser, M.: Beschreibung des Rißgelbietes. - Württ. Min. d. Innern, Bericht d. Straßen- u. Wasserbauverwaltung, 1921.

Ge ologische Úbersich ts kartierung Südwürttembergs unter Leitung von G. WAGNER, 1946-49: die Blätter Saulgau (F. WENK), Munderkingen und Uttenweiler (HeinzelmanN), Schwendi, Warthausen und Laupheim (H. Graul), Aitrach (K. SchëDEL u. a.).

Graul, H.: Untersuchungen über Abtragung und Aufschüttung im Gebiet des unteren Inn und des Hausruck. - Mitt. geogr. Ges. München 1937. - Zur Morphologie der Ingolstädter Ausräumungslandschaft. - Forsch. z. deutschen Landeskunde 43, 1943.

Graul, H., Weidenbach, F. \& Schaefer, I.: Quartärgeologische Exkursion durch die RißLechplatte. - Geol. Bavar. Nr. 6, München 1951.

GrIPP, K.: Über den morphologischen Nachweis großer Schwankungen des Eisrandes. Eiszeit u. Gegenwart 1, S. 65. 1951.

Krauss, K.: Über die sogenannten Deckenschotter des Rißtals. - Jber. u. Mitt. oberrhein. geol. Ver. Bd. 21, S. 66. 1932.

Penck, A. \& BrǘCKNer, E.: Die Alpen im Eiszeitalter. 1901/09.

Poser, H. \& HövermanN, J.: Untensuchungen zur pleistozänen Harzvergletscherung. Abh. braunschw. wiss. Ges. 3, S. 61-115. 1951.

Rathjens, C.: Über die Zweiteilung der Würmeiszeit im nördlichen Alpenvorland. Pet. Mitt. 1951, S. 89-97.

SсHÄDEL, K.: Untersuchungen über das Altdiluvium des Rheingletschers zwischen Iller und Bodensee. Diss. Tübingen 1950 (Manuskript).

SchaEfER, I.: Die Würmeiszeit im Alpenvorland zwischen Riß und Günz. - Augsburg 1940. - Die diluviale Erosion und Akkumulation. - Forsch. z. deutschen Landeskunde 49, 1950.

SснміDт, M.: Geolog. Spezialkarte von Württemberg 1:25000, Blatt Sigmaringen, Stuttgart 1935.

Schreiner, A.: Die Entstehung des Wurzacher Beckens. - Diss. Tübingen 1950 (Manuskr.).

Trols, C.: Bericht über die Deuqua-Tagung 1950 in München. - Erdkunde 1951, S. 86.

WeIDENBACH, F.: Geolog. Spezialkarte v. Württemb. $1: 25000$, Blätter Ochsenhausen u. Biberach. Stuttgart 1937. - Bildungsweise und Stratigraphie der diluvialen Ablagerungen Oberschwabens. - N. Jb. f. Min. usw. Beil. Bd. 78, Abt. B. 1937. - Geologische Karte von Oberschwaben 1:100000, Blatt Ulm. - Stuttgart 1951 (im Erscheinen begriffen).

\section{Die Entstehung der Seen in den ehemals vergletscherten Gebieten}

\author{
Von Paul Woldstedt. Mit $3 \mathrm{Abb}$.
}

I.

In früheren Arbeiten $(1923,1926)$ habe ich die Ansicht vertreten, daß die Hohlformen der Rinnenseen usw. in den Randgebieten der ehemals vergletscherten Gebiete im wesentlichen durch Schmelzwasserströme unter dem Eis gebildet worden seien. Ich stützte mich dabei hauptsächlich auf Arbeiten von N. V. Ussing (1903) und E. Werth (1907, 1909, 1914). Der zuerstgenannte Autor zeigte bei der Untersuchung der großen jütischen Heideebenen, daß diese Sanderflächen der Letzten Vereisung sich aus einzelnen, deutlich individualisierten Schwemmkegeln zusammensetzen. An der Spitze dieser Kegel liegt das gröbste Material; nach auswärts nimmt die Korngröße ab. Die Lage der Kegelspitzen ist dadurch charakterisiert, daß hier die Endmoräne unterbrochen ist und an 
diesen Unterbrechungen Rinnentäler ausmünden, die sich vielfach weithin im ehemals eisbedeckten Gebiet verfolgen lassen.

Die Bezeichnung dieser Täler im Schrifttum wechselt. Im Dänischen werden sie als Tunneltäler bezeichnet. In deutschen Schriften ist von Rinnen- oder Fördentälern die Rede, oft einfach nur von "subglazialen Rinnen“. Es handelt sich um meist über größere Erstreckung hin zu verfolgende, gewöhnlich ziemlich schmale Rinnen, die sich in der Richtung der ehemaligen Eisbewegung erstrekken, und in denen zahlreiche, oft seenerfüllte Wannen hintereinander liegen, die durch Schwellen voneinander getrennt sind. Sie seien hier Rinnentäler genannt.

Untersuchungen in Norddeutschland (WoLDstedT 1913, 1923) zeigten, daß dieselben Beziehungen zwischen Rinnen und Sanderflächen auch für weite Teile des norddeutschen Randgebietes der Letzten Vergletscherung gelten. Große Schmelzwasserströme sind dort aus dem Eisrande ausgetreten, wo Rinnentäler auf den Eisrand stießen. Der Schluß lag nahe, daß die Schmelzwasserströme nicht nur diesen Tälern gefolgt, sondern sie auch ausgefurcht hätten.

In demselben Sinne hatte sich früher schon E. WERTH geäußert. In seiner Arbeit über „Fjorde, Fjaerde und Föhrden“ (1909) schildert er ausführlich die Tunnel- oder Fördentäler und betont besonders ihre Zusammensetzung aus mehreren hintereinander liegenden, jeweils durch Schwellen getrennten Becken. Nach ihm sind es die subglaziären Schmelzwässer gewesen, die diese Formen hervorgebracht haben. „Unter dem Drucke des auf ihnen lastenden Eises waren diese imstande, nicht nur aus der tiefgelegenen Sohle der Föhrde auf die Höhe des Sanderkegels vor dem Eisrande aufwärts zu fließen, sondern auch die subglazialen Rinnen im wesentlichen selbst auszufurchen und ihnen die Eigentümlichkeiten $\mathrm{zu}$ verleihen, die den von subaerisch sich bewegenden Flüssen geschaffenen Tälern fehlen. Die subglazialen Schmelzwässer bewegen sich in der Richtung der Druckentlastung dem Eisrande zu; der Verlauf ihrer Betten muß daher, abgesehen von untergeordneten durch die ursprüngliche Oberflächengestaltung bedingten Unregelmäßigkeiten, im großen ganzen der Bewegungsrichtung des Eises entsprechen".

In meiner zusammenfassenden Behandlung der Seenbildung kam ich 1926 zu dem Ergebnis, daß bei einem Teil der Seen (und Förden) der Gletscher selber mit an der Bildung beteiligt gewesen sei, daß also Gletscher- und S chmelzwässer zus a m m enge wirkt hätten. 1929 (S. 86) betonte ich, daß möglicherweise die Gletscher-Erosion die erste Formung der Rinnen hervorgebracht habe, die dann von den Schmelzwässern weiter ausgestaltet seien. Nach weiterer Beschäftigung mit dieser Frage muß ich heute zu der Anschauung kommen, daß die Seen, Rinnen, Förden usw. in ihrer heutigen Form im wesentlichen ein Ergebnis der Gletschererosion sind und daß die Schmelzwässer nur in beschränktem Umfang mitgewirkt haben.

Dafür spricht vor allem die Ausbildung der Rinnentäler selber, und zwar sowohl im Längs- wie im Querprofil. Für das Längsprofil ist, wie schon mehrfach gesagt worden ist, die Zusammensetzung aus einzelnen hintereinander liegenden Becken charakteristisch, die durch Schwellen getrennt sind. Das aber ist die typische Talform, wie wir sie ebenso in allen ehemals vergletscherten Gebirgen antreffen, besonders im Längsprofil der Trogtäler. Dort zweifelt niemand daran, daß es sich um Erosionsformen des Gletschers selber, nicht seiner Schmelzwässer, handelt. 
Ein weiterer Punkt ist noch wichtiger. Die Rinnentäler verjüngen sich normalerweise zum Eisrande hin. Wären die Schmelzwässer wesentlich an der Talbildung beteiligt, so sollte man das Umgekehrte erwarten. Da die Schmelzwässer zum Randgebiete des Gletschers immer mehr zunehmen, dementsprechend immer mehr subglaziäre Schmelzwasserrinnen sich im Randgebiete unter dem Gletscher vereinigen müssen, sollte man annehmen, daß die Rinnentäler zum Eisrande an Breite und Tiefe zunehmen. Gerade das Gegenteil ist der Fall. Die Becken der schleswigschen Förden teilen sich in einzelne Zweigbecken auf, die sich ihrerseits zum Eisrande hin heben, sich verjüngen und am Eisrande aufhören. Es sind dieselben Formen, wie sie auch die voralpinen Stamm- und Zweigbecken zeigen. Es besteht wohl Einigkeit darüber, daß es sich bei diesen um Formen des Eises, nicht um solche der Schmelzwässer handelt.

In Anlehnung an Werth glaubte ich, daß veränderte Druckverhältnisse des Wassers in und unter dem Eis vielleicht zur Erklärung der Formen beitragen könnten. Aber das Wasser steht im Randgebiet des Gletschers nicht „unter dem Druck des darauf lastenden Eises“. Man kann die Wasserverhältnisse im spaltendurchsetzten Randgebiet eines Gletschers oder Inlandeises wohl am ehesten mit denen eines Karstgebietes vergleichen. Im allgemeinen ist ein Karstwasserspiegel vorhanden, bis zu dem die Spalten mit Wasser erfüllt sind. Dabei kann ein einzelnes geschlossenes Kanalsystem davon abweichen. Hier regelt sich der Wasserstand nach dem System der kommunizierenden Röhren. Liegt in einem solchen der Wasserzufluß, z. B. von der Oberfläche des Gletschers her, hoch, so kann das Wasser am Gletscherrande unter Druck austreten und gegebenenfalls aufsteigen. Es können auch beim Herunterstürzen des Wassers Strudellöcher im Untergrund ausgekolkt werden. Aber die Gesamtspur der subglaziären Schmelzwässer wird nicht die Form eines Rinnentales haben. Sondern diese ist nur als Erosionsform des Gletschers selber zu verstehen.

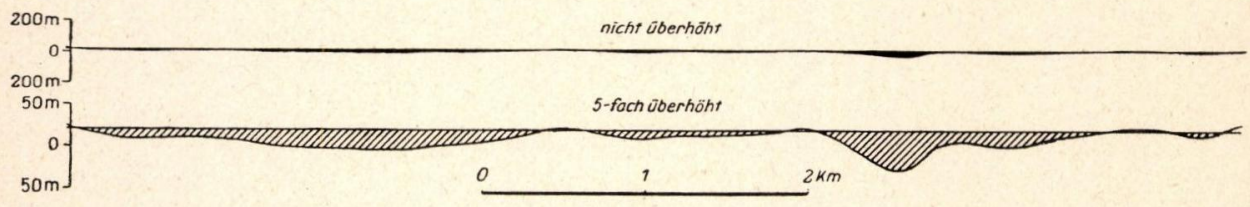

Abb. 1. Querprofil durch den nördlichen Plöner See (Schleswig-Holstein).

$\mathrm{Daß}$ es sich hier nicht um Wasserrinnen, sondern um die Spur des Gletschers handelt, das geht auch aus dem Querprofil hervor. Abb. 1 gibt ein Querprofil durch die besonders tiefe Rinne, die am Boden des Großen Plöner Sees annähernd von $\mathrm{N}$ nach $\mathrm{S}$ zieht. Sie enthält Tiefen bis $60 \mathrm{~m}$. Der Querschnitt durch den nördlichen Plöner See, in WNW-ESE-Richtung geführt nach der Tiefenkarte von G. Wegemann (1922), zeigt, daß noch mehrere flachere Rinnen der Hauptrinne parallel laufen.Im nicht überhöhten Profil ist aber kaum die hier $50 \mathrm{~m}$ tiefe Hauptrinne, geschweige denn die flachen Nebenrinnen zu erkennen. Erst bei fünffacher Überhöhung kommen die Rinnen erkennbar heraus.

Für die Vorstellung von tiefen, durch Schmelzwässer ausgefurchten Erosionsrinnen ist wahrscheinlich in erster Linie die starke Überhöhung verantwortlich, unter der wir gewohnt sind, die Dinge anzuschauen. In Wirklichkeit handelt es sich um ganz schwache Furchungen, die das Eis an seiner Unterfläche hervorgebracht hat. Wenn an Förden gelegentlich steilere Wandungen auftreten, so sind sie, wie auch WeRTH (1909) hervorhebt, meist sekundärer Natur und auf Brandungswirkung und dgl. zurückzuführen. 
II.

Sind nun die Rinnentäler ihrer Form nach keine durch Schmelzwässer ausgefurchten Rinnen, sondern Formen der Gletschererosion, so müßten wir also annehmen, daß sich in ihnen isolierte Gletscherteile bewegt hätten? Diese Annahme ist wohl nur für einzelne von ihnen $\mathrm{zu}$ machen. Im inneren Teil der Apenrader Förde z. B. hat sich zweifellos in einer bestimmten Phase eine Gletscherzunge bewegt. Das geht aus den Endmoränen hervor, die das Fördenende hufeisenförmig umgeben. Gletscherende und Zungenbecken fielen hier zusammen. Aber zweifellos gilt dies nicht für die Mehrzahl der Rinnen im Randgebiet. Diese liegen als radiale Rinnen unter einer im wesentlichen zusammenhängenden, im einzelnen in Loben aufgeteilten Eisdecke. Soll man dann an eine Art linienförmiger Erosion des Eises im Gletscherrandgebiet denken?

Um diese Frage zu beantworten, müssen wir uns ins Alpenvorland begeben, das klassische Gebiet der „Stamm“- und „Zweigbecken“. Solange sich die großen eiszeitlichen Alpengletscher noch innerhalb des Gebirges befanden, erfüllten sie ihr Tal ganz und bis zu beträchtlicher Höhe. Wo sie aber das Gebirge verließen, breiteten sie sich fächerförmig aus. Das eigentliche Tal jedoch, das bisher den ganzen Gletscher enthalten hat, setzt sich unter dem nunmehr weit auseinander gehenden Gletscher noch eine Weile fort, um allmählich an Tiefe und Umfang abzunehmen. Die „Übertiefung“ läuft aus, sagt PENCK. Aber das Stammbecken setzt sich, unter Einschaltung von Schwellen, in einer Reihe von Zweigbecken fort. Stamm- und Zweigbecken zeigen die Formen der Gletschererosion wie die Trogtäler im Gebirge. Wir haben keinen Grund zur Annahme, daß bei den Zweigbecken etwa Schmelzwasser-Erosion vorliegt. Wir müssen also doch eine Art „linienförmiger" Erosion des Gletschers annehmen. Es fragt sich, wie wir uns eine solche vorzustellen haben.

Am besten gehen wir wieder vom Stammbecken aus. Es kann keinem Zweifel unterliegen, daß dort, wo das Stammbecken aus dem Gebirgstal heraustritt, der Gletscher seine größte Eismächtigkeit und Geschwindigkeit hatte. Beides mußte zu einer gegenüber der Umgebung erhöhten Erosion führen, die nach außen hin allmählich abklingt. So erscheint die Ausschürfung eines Stammbeckens durchaus logisch. Schwieriger ist die Erklärung der Zweigbecken. Aber auch hier müssen wir an Zonen stärkerer Erosion denken, und zwar von Teilgletschern. Wenn auch äußerlich noch ein einheitlicher Gletscher vorhanden war, so müssen wir doch annehmen, daß er dynamisch in eine Reihe von Teilgletschern aufgespalten war. Bei jedem Teilgletscher bezeichnet das entsprechende Zweigbecken die Zone stärkster Erosionskraft. Nach der Seite hin nimmt diese ab. Hier liegen - seitlich der Zweigbecken - die Drumlinfelder. Bei ihrer Bildung halten sich Erosion und Akkumulation die Waage. Schließlich folgt randlich die Zone reiner Akkumulation. Es entstehen die Rand- und Ufermoränen, die teilweise als Sporne tief in den Gletscher eindringen, so die Teilgletscher begrenzend. Diese treten immer deutlicher heraus, je geringer die Eismächtigkeit wird.

Warum aber verzweigt sich das Stammbecken nicht einfach fingerförmig in die Zweigbecken? Diese Frage hat auch PENCK nicht befriedigend beantworten können. Wohl ist gelegentlich eine solche fingerförmige Verzweigung vorhanden. Aber meist sind die Zweigbecken durch Schwellen von dem Stammbecken getrennt. Es müssen also bei der Aufgliederung des Eises in Teilgletscher Unterschiede in der Erosionskraft auftreten, wie sie ja auch sonst bei Diffluenzen beobachtet werden. 
III.

Es besteht nun aber der oben dargelegte Zusammenhang zwischen der Mündung der Rinnentäler und den Aufschüttungspunkten der Sanderflächen. Also muß doch Wasser in ihnen geflossen sein. Wie erklärt sich der Gegensatz zwischen der auf Gletschererosion deutenden Form und dem offensichtlichen Austreten von Wasser aus ihnen?

Ich glaube, daß diese Erklärung verhältnismäßig einfach ist. Die vom Gletscher in den Untergrund geschürften Rinnentäler sind die tiefsten Punkte der Gletschersohle. Das gilt insbesondere auch für den Eisrand. Wenn wir die Basisfiäche des Eises entlang seinem Rande verfolgen, sehen wir, daß diese am distalen Ende eines Rinnentals normalerweise am tiefsten liegt. Hier ist der gegebene Punkt für den Austritt der Schmelzwässer, die in den Radialspalten der Gletscherzunge herunterstürzen. So ist also das distale Ende eines Rinnentals als niedrigste Stelle der Gletschersohle am äußeren Gletscherrande die vorgezeichnete Stelle für ein Gletschertor. Bei starker Schmelzwasserwirkung wird das Gletschertor langsam zurückweichen. Es wird in dem dahinterliegenden Teil des Gletschers ein verstärktes Schmelzen einsetzen, allein schon infolge der größeren Wassermengen, die hier hindurchfließen. So kommt es zu einer Einmuldung der Eisoberfläche über dem subglaziären Tal, wie man dies immer wieder an Gletschern beobachten kann. Dabei wächst der Sanderkegel allmählich in den Gletscher hinein.

Der Höhenunterschied zwischen der Sohle des Rinnentals und der Spitze des Sander-Schwemmkegels wird dabei immer größer. Ich nahm mit Ussing und Werth früher an, daß das Wasser diesen Höhenunterschied hydrostatisch überwinden könne, sofern nur die Versickerungsstelle des Wassers auf der Oberfläche des Gletschers höher gelegen habe. Aber diese Annahme macht doch Schwierigkeiten. Wesentlich einfacher ist die Vorstellung, daß der Schmelzwasseraustritt sich selber immer höher legt, d. h. daß die Schmelzwässer z. T. garnicht an der Sohle des Gletschers verlaufen, sondern in Kanälen in und über Eis, das die tieferen Partien der Rinnentäler erfüllt. Das hat auch E. TodmanN (1936) angenommen, ausgehend von Beobachtungen am Vatnajökull auf Island.

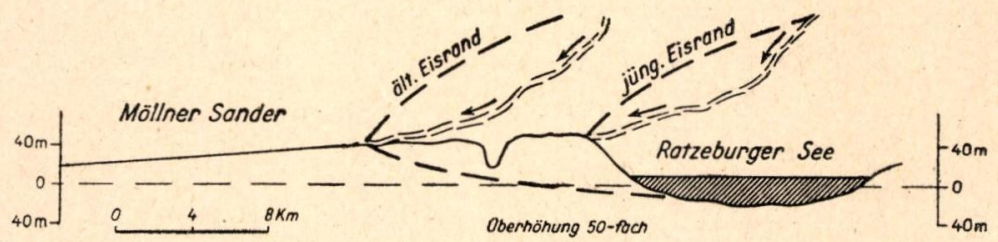

Abb. 2. Längsprofil durch Möllner Sander und Ratzeburger See.

Als Beispiel für das Hineinfressen eines Sanderkeils in die Eisfront und die damit verbundene Höherlegung der Sanderspitze kann der von mir 1926 (S. 110) beschriebene Möllner Sander gelten (vgl. hierzu Abb. 2). Ursprünglich lag der Austrittspunkt der Schmelzwässer südwestlich Gudow. Allmählich wurde er um etwa $15 \mathrm{~km}$ nach Norden zurückverlegt. Dabei stieg seine Höhe um mehr als $10 \mathrm{~m}$. Die ältere und die jüngere Lage des Eisrandes sind in Abb. 2 angedeutet, ebenso der mutmaßliche Verlauf der Schmelzwässer im Eis. Die in der älteren Mölln-Gudower Rinne liegen gebliebenen Toteisreste wurden dabei übersandet und waren zunächst völlig zugedeckt. Erst viel später, wahrscheinlich erst zu Beginn der Holozäns, schmolzen die Toteisreste allmählich im Untergrund aus, 
und die alte Rinne mit mehreren hintereinader liegenden Wannen trat wieder in Erscheinung (vgl. Abb. 8 in WoLDSTEDT 1926).

IV.

Zur Klärung der in Abschnitt III geäußerten Anschauungen führen weitere Beobachtungen im Randgebiet der Nordeuropäischen Vergletscherung. Schon mehrfach (K. Milthers 1935, P. Woldstedt 1938) ist darauf hingewiesen worden, daß die verschiedenen Randlagen des Inlandeises teils mehr als Vorstoß-, teils mehr als Abschmelzfronten entwickelt sind. Die Vorstoßfronten zeigen uns den noch nicht erheblich veränderten Zustand, wie ihn der Eisrand nach einem kräftigen raschen Vorstoß aufwies. Wahrscheinlich ging hinterher der aktive Eisrand verhältnismäßig schnell zurück. So wurden die Formen der Vorstoßfront nur ziemlich wenig gestört. Die Abschmelzfronten dagegen sind das Ergebnis einer längeren Zeit, während der der Eisrand sich annähernd in derselben Zone hielt.

Es zeigen sich nun, was die Rinnentäler anbelangt, charakteristische Unterschiede zwischen den Vorstoß- und den Abschmelzfronten. Im wesentlichen eine Vorstoßfront ist z. B. das Pommersche Stadium. Bei diesem beobachten wir eine ganz ähnliche Ausbildung der Stamm- und Zweigbecken wie im Alpenvorland. Darauf hat schon E. WERTH (1917, S. 115) hingewiesen. Abb. 3 zeigt, in Anlehnung an E. Werth, das Gebiet des Oderlobus. Als Stammbecken muß das Haffgebiet angesehen werden, das heute zum größten Teil durch jüngere Ablagerungen zugefüllt ist. Von diesem Stammbecken gehen die Zweigbecken aus. Die wichtigsten sind: die Prenzlauer Rinne, das Randowtal, das Odertal und die Plöne-Rinne. Das Randow- und das Odertal sind, als spätglaziale Abflußrinnen, stark umgestaltet worden. Sie zeigen keine hintereinander liegenden, durch Schwellen getrennte Becken mehr. Wohl aber enthalten solche die beiden anderen Zweigbecken. In der Prenzlauer Rinne liegen hintereinander der Untere und der Obere Ücker-See. Im Plöne-Zweigbecken liegen ähnlich hintereinander

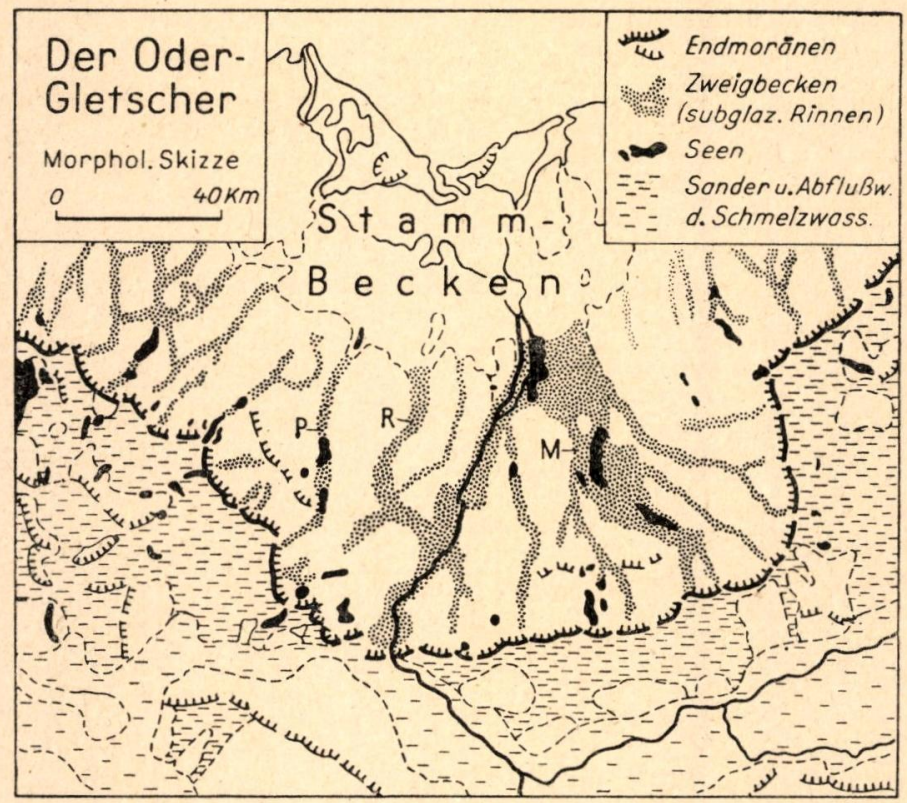

Ab. 3. Morphologische Skizze des Odergletschers während des Pommerschen Stadiums der Letzten Vereisung. $\mathbf{P}=$ Prenzlauer, $\mathrm{R}=$ Randow-, $\mathbf{M}=\mathbf{M}$ Mad̈̈-Plöne-Zweigbecken. 
die Wannen des Madü- und Plöne-Sees. Das Plöne-Zweigbecken zeigt ausgeprägt die zum Eisrande hin sich verjüngende Form der subglaziären Täler.

Wo aber die Plöne-Rinne auf den Eisrand trifft, ist dieser leicht eingebuchtet, und in diese Einbuchtung legt sich der Gipfelpunkt des Berlinchener Sanders (vgl. Woldstedt 1923) hinein. Große Schmelzwasserfluten müssen hier ausgetreten sein. Aber dies bezeichnet wahrscheinlich schon einen gegenüber dem ersten Vorstoß etwas vorgeschrittenen Zustand. Ursprünglich mag hier der Eisrand kaum eingebuchtet gewesen sein. Es lag aber hier die tiefste Stelle der Eisunterkante im Zuge des Eisrandes, und das war der Punkt, an dem am leichtesten Schmelzwässer austreten konnten. Dies führte in der schon geschilderten Form allmählich zu einer Einmuldung der Eisoberfläche und damit zu einer weiteren Verstärkung der Schmelzwässer in diesem Gebiet. Der Sander begann sich in den Eisrand hineinzufressen.

Aber beim Berlinchener Sander beobachten wir nur den ersten Anfang dieser Entwicklung. Die tief eingreifenden Sanderbuchten, wie sie beim Brandenburger und Frankfurter Stadium vorhanden sind, fehlen dem Pommerschen. Bei diesem treten überhaupt die eigentlichen Kegelsander zurück gegenüber der allgemeinen Sanderbildung am Eisrande. Ganz anders beim Brandenburger und Frankfurter Stadium. Bei ihnen finden wir die tief in den Eisrand sich hineinfressenden Kegelsander, wie etwa den Beelitzer, den Schweriner, den Möllner und den Bornhöveder. Wir müssen sie auffassen als das Ergebnis vorgeschrittener Abschmelzung. Mit ihr geht eine charakteristische Umgestaltung des Eisrandes vor sich. Der Eisrand an der Stirn der Zweigbecken, der ursprünglich nicht eingebuchtet, vielleicht sogar leicht vorgewölbt war, buchtete sich jetzt unter dem Einfluß der Schmelzwässer mehr und mehr ein, bis schließlich die spitz keilförmig in das Eis eingreifenden Sander entstanden. Solche Kegelsander charakterisieren die Abschmelzfronten, während eine mehr gleichmäßig vor dem Gletscherrand entwickelte Sanderzone für die Vorstoßfronten charakteristisch ist.

Wir haben bisher die Entwicklung der Gletschertore vom Maximum eines Vorstoßes bis zum Zerfall des Eises betrachtet. Aber auch beim Vorrücken des Eises bis zum Maximum müssen Schmelzwässer gebildet und durch Gletschertore abgeflossen sein. Mit dem vorrückenden Gletscher sind auch die Gletschertore vorgerückt, wenn sie auch z. T. ihre Lage wechselten. Sie waren normalerweise auch damals die tiefsten Punkte des Gletscherrandes. Man könnte sich vorstellen, daß vielleicht einzelne Zweigbecken als die Spuren der vorwärtsschreitenden Gletschertore aufzufassen seien. Sie wurden vom nachfolgenden Eis, das in sie hineindrang, überformt, und so bekamen diese Zweigbecken ihre heutige Form. Trifft diese Anschauung zu, so wäre das Schmelzwasser, das im Gletschertor austrat, zunächst die Säge gewesen, mit der ein Einschneiden in den Untergrund erfolgte. Die weitere Ausgestaltung der Rinnen geschah dann durch das nachrückende Gletschereis.

V.

Die Formen der Rinnentäler sind, wie ausgeführt wurde, nicht mit der Vorstellung zu vereinigen, daß sie allein durch Schmelzwässer gebildet sein könnten. Bei einigen mag die erste Anlage durch Schmelzwässer vorgezeichnet sein. In ihrer heutigen Form sind sie aber in der Hauptsache Erosionsformen des Eises selber (die im Mündungsgebiet wiederum etwas durch Schmelzwässer umgestaltet sein können). Für diese Auffassung spricht weiter, daß dieselben Formen auch in den zentralen Ausräumungsgebieten der ehemaligen Inlandeise auftreten. Hier sind diese Formen in den anstehenden Fels hineingeprägt worden: In der Richtung der Eisbewegung verlaufende Rinnen mit einzelnen hinterein- 
ander liegenden, durch Schwellen getrennten Becken. Das ist in Kanada ebenso der Fall wie in Fennoskandien.

Das schönste Bild eines solchen in den Felsen geschnittenen Rinnenfächers bietet Finnland. Die Haupterstreckung der Seen geht von NW nach SE., d. h. sie folgt der Hauptbewegungsrichtung des Eises. J. Sederholm (1913) allerdings wollte einen Zusammenhang erkennen zwischen der Erstreckung der Seen und den Spaltensystemen des Untergrundes. Nach seiner Ansicht hätte der Gletscher nur ausräumend gewirkt. Aber wir finden dasselbe Bild in allen ehemals vergletschert gewesenen Gebieten: überall im Wesentlichen Übereinstimmung der Seenrichtung mit der Eisbewegung, wie sie durch die Gletscherschrammen angedeutet wird (vgl. auch das Bild des südschwedischen Seenfächers bei WERTH 1909). Das spricht für unmittelbare, in der Richtung der Eisbewegung erfolgte Eiserosion, wobei im einzelnen der Anlaß für eine Erosion sehr verschieden sein mag.

Von den schmalen, in der Richtung der Eisbewegung liegenden Rinnenseen finden sich alle Übergänge zu breiteren, zungenbeckenartigen Formen. Hierher gehören die großen alpinen und westpatagonischen Randseen, schließlich die gewaltigen nordamerikanischen Seen - besonders typisch der Michigan-See und die Ostsee. Sie alle sind Formen des ausschürfenden Eises. Bei diesen haben die Schmelzwässer keine wesentliche Rolle gespielt. Das haben sie nur bei einem Teil der Rinnen im Gletscherrandgebiet getan, wo sie gelegentlich die Rinnen etwas umgeformt haben. Auf diese Umformungen soll in einer späteren Arbeit näher eingegangen werden.

$\mathrm{Daß}$ die Erhaltung der Hohlformen meist ihrer Füllung mit Toteis zu verdanken ist, das die Becken vor späterer Zuschüttung bewahrte, das ist an so zahlreichen Beispielen im einzelnen nachgewiesen worden, daß hier nicht mehr darauf eingegangen zu werden braucht. Merkwürdig spät erst ist man für die Alpenrandseen zu dieser Anschauung gelangt. Aber zweifellos hat R. STAub (1938) recht, wenn er auch für die Erhaltung der alpinen Randseen in weitestem Maße Toteis annimmt. Ob dabei wirklich die Lage in heute föhnfreien Gebieten entscheidend ist, wie STAUв annimmt, bedarf wohl noch näherer Untersuchung.

$\mathrm{Sch}$ if ten - N a ch weis

Milthers, K.: Landskabets Udforming mellem Alheden og Limfjorden. - Danm. Geol. Unders. II. R. No. 56. Kopenhagen 1935.

Penck, A. \& BrÜCKner, E.: Die Alpen im Eiszeitalter. - Leipzig 1901-09.

Sederholm, J.: Weitere Mitteilungen über Bruchspalten. - Bull. Com. Géol. Finlande $37,1913$.

Staub, R.: Prinzipielles zur Entstehung der alpinen Randseen. - Ecl. Geol. Helv. 31, S. 239-258. 1938.

Todtmann, E. M.: Einige Ergebnisse von glazialgeologischen Untersuchungen am Südrand des Vatna-Jökull auf Island. - Z. deutsch. geol. Ges. 88, S. 77-87. 1936.

Ussing, N. V.: Om Jyllands Hedesletter og Teorierne om deres Dannelse. - Overs. k. danske Vid. Selsk. Forh. 1903.

Wegemann, G.: Die Seen Ostholsteins. - Kiel (W. G. Mühlau) 1922.

WERTH, E.: Studien zur glazialen Bodengestaltung in den skandinavischen Ländern. Z. Ges. Erdk. Berlin 1907. - Fjorde, Fjaerde und Föhrden. - Z. f. Gletscherk. 3, 1909. - Zur Oberflächengestaltung der südschwedischen Halbinsel. - Ebendort 8, 1914. - Das Eiszeitalter. 2. Aufl. Berlin (Göschen) 1917.

Woldstedt, P.: Beiträge zur Morphologie von Nordschleswig. - Mitt. geogr. Ges. Lübeck (2) 26, S. 41-110. 1913. - Studien an Rinnen und Sanderflächen in Norddeutschland. - Jb. preuß. geol. L.A. 42, S. 780-820. 1923. - Probleme der Seenbildung. - Z. Ges. Erdk. Berlin, S. 103-124. 1926. - Das Eiszeitalter. Stuttgart (F. Enke) 1929. - Über Vorstoß- und Rückzugsfronten des Inlandeises in Norddeutschlnad. - Geol. Rundschaiu 29, S. 481-490. 1938. 